\title{
The SUMOylation landscape of renal cortical collecting duct cells
}

\author{
Qi Wü, Takwa S. Aroankins", Lei Cheng, Robert A. Fenton* \\ InterPrET Center, Department of Biomedicine, Aarhus University, Aarhus DK- \\ 8000, Denmark \\ "These authors contributed equally to this work
}

* Correspondence:

Robert A. Fenton

Aarhus University

Department of Biomedicine

Wilhelm Meyers Allé 3, Building. 1233

DK- 8000 Aarhus C

Denmark

Tel: +4587167671

E-mail: robert.a.fenton@biomed.au.dk 


\section{ABSTRACT}

Protein post-translational modification by the small-ubiquitin-related-modifier (SUMO) is a mechanism that allows a diverse response of cells to stress. Five SUMO family members, SUMO1-5, are expressed in mammals. We hypothesized that because kidney epithelial cells are often subject to stresses arising from various physiological conditions, multiple proteins in the kidney will be SUMOylated. Here we profiled SUMO1 and SUMO2 modified proteins in a polarized epithelial cell model of the renal cortical collecting duct (mpkCCD14 cells). Modified forms of SUMO1 or SUMO2, with a histidine tag and a Thr to Lys mutation preceding the carboxyl-terminal di-gly motif were expressed in mpkCCD14 cells allowing SUMO-conjugated proteins to be purified and identified. Protein mass spectrometry identified 1428 SUMO1 and 1957 SUMO2 sites, corresponding to 741 SUMO1 and 971 SUMO2 proteins. Gene ontology indicated that the function of the majority of SUMOylated proteins in mpkCCD14 cells was related to gene transcription. After treatment of the mpkCCD14 cells for $24 \mathrm{~h}$ with aldosterone, the levels of SUMOylation at a specific site on the proton and oligopeptide/antibiotic cotransporter protein Pept2 were greatly increased. In conclusion, the SUMOylation landscape of mpkCCD14 cells suggests that protein modification by SUMOylation is a mechanism within renal epithelial cells to modulate gene transcription under various physiological conditions.

KEYWORDS: Posttranslational modification, kidney, proteomics, ion transport, epithelia 


\section{INTRODUCTION}

The Small Ubiquitin-like MOdifier (SUMO) is a covalent protein post-translational modification (PTM) that alters the function of target proteins and cell processes including DNA/RNA transcription, RNA translation, DNA repair, nucleocytoplasmic transport, apoptosis and protein stability ${ }^{1}$. Furthermore, protein SUMOylation increases under conditions of cell stress, including osmotic, genotoxic and oxidative stresses ${ }^{2}$. Five SUMO family members, SUMO1-5, are expressed in mammals, whereas SUMO1-3 are expressed in most species ${ }^{3-5}$. SUMO2 and SUMO3 share a high degree of sequence homology ${ }^{2}$ SUMO is conjugated onto a target lysine (K) by an enzymatic cascade involving a dimeric E1 activating enzyme, a single E2 conjugating enzyme (Ubc9) and several E3 SUMO protein ligases. SUMO target site recognition and subsequent SUMOylation occurs by three different, but not mutually exclusive mechanisms: a) target proteins can contain a SUMO consensus motif (mainly $\Psi$ KXE) that can be recognized directly by Ubc9; b) target proteins contain a SUMO interaction motif (SIM) that recruits the SUMO-Ubc9 complex or; c) the target protein has an E3-ligase interaction site that aligns the SUMO-Ubc9-E3 complex to the specific K-residue to be SUMOylated - forming a di-glycinelysine bond with the targeted protein (K-GG). SUMOylation is a reversible process, with SUMO specific proteases (sentrin specific proteases, SENP) deSUMOylating target proteins ${ }^{6-8}$. Rapid conjugation and de-conjugation of SUMO allows cells to dynamically adjust to a wide span of cellular stresses ${ }^{9}$.

Over the past decade, tandem liquid chromatography coupled mass spectrometry (LCMS/MS)-based proteomics and bioinformatics has enabled rapid and high-throughput discovery of novel PTM sites ${ }^{10}$. As SUMOylation is a rare modification ${ }^{11}$, many of the methods for SUMO proteomic analysis involve using expression of an epitope-tagged SUMO to facilitate protein 
enrichment ${ }^{9}$. Furthermore, mutations of the expressed SUMO are required to produce a shorter peptide chain after SUMO cleavage by trypsin that is compatible with LC-MS/MS analysis ${ }^{12}$. Additional methods for purification and detection of SUMOylation by LC-MS/MS have also been recently developed ${ }^{11,13-17}$.

Currently there have been few investigations of the role of protein SUMOylation in the kidney. Of the current studies, SUMOylation appears to play a cytoprotective role in acute kidney injury ${ }^{18}$ and reduce progression of renal cell carcinoma ${ }^{19}$, whereas increased SUMOylation has been linked to renal fibrosis and the progression of diabetic nephropathy and podocyte injury processes ${ }^{20}$. The lack of studies regarding SUMOylation in the kidney is surprising considering the tight hormonal control of kidney function, plus the various cellular stresses, including osmotic and oxidative, that kidney epithelial cells are routinely subject to. Therefore, the aim of this study was to generate a SUMOylation landscape of a polarized epithelial cell model of the renal cortical collecting duct, mpkCCD14 cells, as a first step to understand the role of SUMOylation in the kidney under physiological and pathophysiological conditions.

\section{MATERIALS AND METHODS}

A complete and detailed description of the methods is enclosed in Supplemental Information.

Cell culture, transfection and antibodies. Mouse kidney cortical collecting duct cells (mpkCCD14) were cultured as described ${ }^{21}$. Cells were stably transfected using standard protocols for Lipofectamine 2000 (Invitrogen), with pEFIRESpuro vectors containing N-terminal hexa-histidine tagged SUMO1 (6xHis-SUMO1 ${ }^{\text {T95K }}$ ) and SUMO2 (6xHis-SUMO2 $\left.{ }^{\text {T9OK }}\right)^{14}$. For mouse Pept2 (S/c15a2) 
studies, mpkCCD14 cells were transfected with flag-tagged mouse Pept2 in a pcDNA3.1(+)-NDYK vector (GenScript). Stably expressing cells were selected using $2 \mu \mathrm{g} / \mathrm{ml}$ puromycin (SigmaAldrich) and named mpkCCD-SUMO1 $1^{\text {T95K }}$ or mpkCCD-SUMO2 ${ }^{\text {T90K }}$ cells. For Pept2 transfected cells $0.15 \mu \mathrm{g} / \mathrm{ml}$ Geneticin (cat\# 10131-019, ThermoFisher) was used. Antibodies utilized were against SUMO1 (cat\# AM1200a, Abgent), SUMO2 (cat\# AM1224a, Abgent), 6 x His (cat\# A00186, GenScript), FLAG-tag (cat\# F7425, Sigma-Aldrich), Proteasome 20s (cat\# sc67339, Santa Cruz) and $\alpha$-actin (cat\# A2066, Sigma-Aldrich).

Cell experiments. mpkCCD-SUMO1 ${ }^{\mathrm{T} 95 \mathrm{~K}}$ or mpkCCD-SUMO2 ${ }^{\mathrm{T} 90 \mathrm{~K}}$ cells were cultured as described ${ }^{22}$. In general, four T175 flasks were required to yield 60-90 mg of crude protein lysates for affinity purification. For heat shock studies, cells were subjected to $1 \mathrm{~h}$ at $43^{\circ} \mathrm{C}$ in a standard incubator. For other experiments, cells were treated with $10 \mu \mathrm{M}$ MG132 (Sigma-Aldrich) for five hours, with the last hour also containing $22 \mu \mathrm{M}$ PR619 (Abcam). SILAC experiments were performed as described $^{22}$, then switched to experimental media (standard media with addition of HEPES, DGlucose and respectively light or heavy amino acids) for $48 \mathrm{~h}$. Cells were treated for $24 \mathrm{~h}$ with either $1 \mu \mathrm{M}$ aldosterone (light labelled cells) or DMSO vehicle (heavy labelled cells).

Real-time quantitative polymerase chain reaction (RTqPCR). Cells transfected with a plasmid encoding mouse Pept2 cDNA were treated with aldosterone or DMSO as described above. RNA extraction and RTqPCR were performed as previously described ${ }^{23}$. Primer pairs utilized were: forward 5'- ACA CAG GAA TCA AAC CAG CCA-3', reverse 5'-CCA TAG TCT TTG CCC ACG CT-3'. Data was analyzed using an unpaired students t-test using GraphPad Prism 7. A p-value $<0.05$ was considered significant.

Immunocytochemistry and confocal laser scanning microscopy. Cells were cultured on glass coverslips until confluent. For experiments with aldosterone, experiments were similar to as 
described above. Immunolabeling was performed as previously described ${ }^{24}$ using 6 x His tag (at 1:5000, cat\# MA1-135, ThermoFisher), SUMO1 (at 1:100, cat\# AM1200a, Abgent) and SUMO2 (at 1:250, cat\# AM1224a, Abgent) antibodies.

Affinity purification with $\mathrm{Ni}^{2+}$-coated agarose beads. Cells were lysed in customized lysis buffer. Approximately $60 \mathrm{mg}$ of protein lysate was mixed with $800 \mu \mathrm{l}$ HisPur $^{\mathrm{TM}}$ Ni-NTA Resin (ThermoFischer) in a $10 \mathrm{ml}$ spin column overnight (maximum $18 \mathrm{~h}$ ) at $4^{\circ} \mathrm{C}$ with rotation. Beads were washed three times in wash buffer 1 , followed by two washes in wash buffer 2 and finally two washes in wash buffer 1 . Proteins were eluted using successive 20 minute incubations with $1 \mathrm{ml}$ elution buffer. Eluates were combined before subsequent processing.

Filter-aided sample preparation (FASP). Ni-NTA purified proteins were loaded onto Vivacon 500, 30kD cut-off spin columns (Sartorius), and washed three times with $500 \mu \mathrm{l}$ UA buffer. Proteins were incubated for one hour at $56^{\circ} \mathrm{C}$ with $50 \mathrm{mM}$ DTT in UA buffer followed by incubation in the dark for 20 minutes at room temperature with $50 \mathrm{mM}$ 2-chloroacetamide in UA buffer. Following centrifugation, filters were washed with IAP buffer and proteins digested overnight at $37^{\circ} \mathrm{C}$ with 1:50 lysyl endopeptidase (Wako Pure Chemical Corporation) in IAP buffer. Peptides were collected by centrifugation (Lys-C digested peptides) and the remaining larger peptides on the filters were subjected to digestion with 1:100 glycyl endopeptidase (ThermoFischer) at $25^{\circ} \mathrm{C}$ overnight (Lys-C + Glu-C digested peptides).

K-GG immunoprecipitation (KGG-IP). Peptides were purified using a KGG-IP kit (Cell Signaling Technology) as described ${ }^{14}$. Briefly, the beads washed three times and then samples incubated overnight at $4^{\circ} \mathrm{C}$ with end-over-end mixing. Beads were collected by centrifugation and washed. Finally, the antibody purified peptides were extracted with $0.15 \%(\mathrm{v} / \mathrm{v})$ TFA. The eluate was vacuum dried before LC-MS/MS analysis. In one batch of cells, Lys-C peptides were subjected to 
high-pH peptide fractionation. These separate fractions were subjected to K-GG immunoprecipitation.

Nano-liquid chromatography and tandem mass spectrometry (LC-MS/MS) and data analysis. Purified peptides were subjected to LC-MS/MS analysis. Q-Exactive settings (ultra-sensitive) were a loop count of 1 , and a maximum injection time of $1000 \mathrm{~ms}$. Sites profiling data was analyzed by MaxQuant (version 1.5.5.1). All the raw files from one SUMO family (SUMO1 or SUMO2) were searched together.

MS data analysis for SILAC experiment. Data were searched with Proteome Discoverer (v2.1.0.81) with both Sequest and Mascot (v2.5). Two parallel processing nodes, corresponding to LysC and LysC+GluC digest were set up. Settings were highly similar to MaxQuant search, with modifications related to SILAC. The mass spectrometry proteomics data for SUMO profiling have been deposited to the ProteomeXchange Consortium via the PRIDE ${ }^{25}$ partner repository with the dataset identifier PXD013346, and the SILAC data with the dataset identifier PXD013363.

Bioinformatics. Motif analysis. Motif analysis was done by Sequencelogo (https://www.phosphosite.org/sequenceLogoAction). G-protein coupled receptor (GPCR), kinase, transcription factor (TF), E3 ligase, deubiquitinating enzyme (DUB), transporter and channel analysis. The identified SUMO proteins from mpkCCD14 cells and ubiquitylated proteins from mouse kidney ${ }^{26}$ were cross-referenced with online databases, to categorize proteins. Databases $\begin{array}{llll}\text { used } & \text { were: } & \text { Association } & \text { Database }\end{array}$ (https://zhanglab.ccmb.med.umich.edu/GLASS/); $\quad$ Mouse KinBase (http://kinase.com/web/current/kinbase/genes/SpeciesID/10090/fasta/protein/); Animal Transcription Factor DataBase (TFDB) $2.0^{28}$ (http://bioinfo.life.hust.edu.cn/AnimalTFDB2/); 
Ubiquitin and Ubiquitin-like Conjugation Database ${ }^{29}$ (UUCD, http://uucd.biocuckoo.org/); transporter and channel $\left.\right|^{30}$ (https://hpcwebapps.cit.nih.gov/ESBL/Database/NephronRNAseq/Transporters and Channels. html). All curated databases used and extracted from these sources are provided in Table S1. STRING analysis. Protein-protein interactions between SUMOylated transcription factors (TFs) and known water and electrolyte transporters/channels ${ }^{30}$ were assessed using STRING ${ }^{31}$ (version 10, https://string-db.org/). Interactions with at least medium confidence (interaction score $\geq 0.4$ ) between a TF and a transporter were retained. Clue GO analysis. SUMO1 and SUMO2 unique and shared proteins were assessed using Clue GO. Ingenuity Pathway Analysis (IPA). SUMO1 and SUMO2 unique proteins were assessed individually using core analysis with default parameters. The two core analysis results were subjected to comparison analysis to generate heatmaps.

\section{RESULTS}

Development and verification of SUMO1 and SUMO2 expressing mpkCCD14 cells. To study SUMOylation events by LC-MS/MS, mpkCCD14 cells were stably transfected with $6 x$ HisSUMO1 $^{\text {T95K }}$ and $6 \mathrm{xHis-SUMO2}{ }^{\text {T90K }}$ constructs $^{14}$. Compared to mpkCCD14-WT cells (Figure S1),

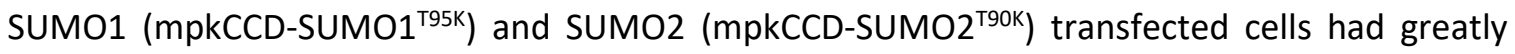
increased levels of the free SUMO moiety of $\sim 13 \mathrm{kDa}$ and a broad smear representing a range of SUMOylated proteins. Protein SUMOylation can be increased in cells by inhibition of deSUMOylation enzymes (PR619), the proteasome (MG132), or by heat shock treatment ${ }^{13,14}$. Treatment of all the cell lines demonstrated a modest increase in SUMOylated proteins (as represented by the smeared signal on western blots) following MG132/PR619 treatment, 
whereas heat shock greatly depleted free SUMO and increased the abundance of SUMOylated proteins (Figure 1A-C). As treatment-induced changes in SUMOylated proteins could be observed in mpkCCD14-WT cells, it is likely not an artifact of the SUMO overexpression. To examine the localization of SUMO in polarized epithelial cells, immunocytochemistry was performed. In both the mpkCCD14-WT and mpkCCD-SUMO1 ${ }^{\text {T95K }}$ cells under control conditions, although SUMO1 was detected in the cytoplasm and the nucleus, it was predominantly identified around the cell nucleus, perhaps represents labeling of proteins or free SUMO in the nuclear envelope (Figure 1D-E). In mpkCCD14-WT and mpkCCD-SUMO2 ${ }^{\text {T90K }}$ cells, SUMO2 labeling was perinuclear but also dispersed throughout the cytoplasm. A similar labeling pattern using antibodies targeting $6 \mathrm{xH}$ is (in the transfected lines) or the native SUMO proteins solidifies these observations. In contrast to control conditions, following heat-shock SUMO1 and SUMO2 labeling was predominantly within the nucleus, with some perinuclear labeling observed (Figure 1D-E). This nuclear localization of SUMO1 and SUMO2 in these conditions matches previous studies $^{32,33}$. Taken together, these results suggest that in polarized renal epithelial cells SUMO is distributed differently under basal or stress conditions.

Affinity isolation of SUMOylated proteins for LC-MS/MS analysis. mpkCCD-SUMO1 $1^{T 95 K}$ or mpkCCD-SUMO2 ${ }^{\text {T90K }}$ cells were treated with MG132 / PR619 or heat shock and subjected to nickel affinity chromatography. After protocol optimization, including alterations of wash buffer salt, imidazole and 2-mercaptoethanol concentrations, SUMOylated proteins could be efficiently isolated from crude protein lysates (Figure $\mathbf{2 A}$ ). These eluates were digested with Lys-C and GluC using Filter-Aided Sample Preparation (FASP) ${ }^{34}$, immunoaffinity enriched for SUMOylated peptides using a K- $\varepsilon-G G$ antibody and subjected to LC-MS/MS analysis using ultra-sensitive 
settings ${ }^{14}$ to maximize SUMOylated peptide identification. About half of the identified peptides and proteins contained SUMO sites (Table S2). The general workflow for all the steps in this study is illustrated in Figure 2B.

SUMO1 and SUMO2 share some similar target proteins in mpkCCD14 cells. 1428 SUMO1 sites, corresponding to 741 SUMOylated proteins were identified in mpkCCD-SUMO1 ${ }^{\text {T95K }}$ cells (Figure 3A and B, Table S3). In mpkCCD-SUMO2 ${ }^{\text {T90K }}$ cells, 1957 SUMO2 corresponding to 971 SUMOylated proteins were detected. Comparisons between the data indicated that approximately $40 \%$ of the SUMOylation sites and $50 \%$ of SUMOylated proteins are targeted by both SUMO1 and SUMO2 (Figure 3C and D). The majority of SUMOylated proteins identified had only one SUMO site, with the percentages of single- and multi-SUMOylated proteins highly similar between SUMO1 and SUMO2 (Figure 3E and F). A comparison of the SUMO2 sites/proteins identified in this study with a recent study where endogenously SUMOylated proteins were identified in the kidney ${ }^{17}$ demonstrated a potential complementary nature of different methods to study SUMO targets (Figure 3G and H), with different SUMO sites identified with the different methodology. Motif analysis by revealed a classic KxE consensus motif and a reversed KxD/E motif (Figure 3I). The potential for SUMO-phosphorylation comodification (presence of the two PTMs within a short amino acid sequence) was determined by assessing all the known phosphorylation sites from PhosphoSitePlus (https://www.phosphosite.org/homeAction) (Figure 3J) or only phosphorylation sites identified in this study (Figure 3K) in relation to the SUMOylation sites. When all known phosphorylation sites were considered, phosphorylation events surrounding SUMOylation sites were evenly distributed, however, when only phosphorylation sites identified in this study were taken into 
consideration, we saw an uneven occupancy where +5 and -5 sites showed predominance. This was partly observed in other studies ${ }^{13,17}$ and may indicate that SUMOylation is partly dependent on phosphorylation events ${ }^{35}$.

\section{Majority of SUMOylated proteins in mpkCCD14 cells are related to control of gene expression.}

Gene ontology (GO) analysis highlighted that proteins SUMOylated by SUMO1 or SUMO2 were associated to different cellular functions. Proteins SUMOylated by only SUMO1 were predominantly associated with histone modification (Figure 4A), whereas those targeted by only SUMO2 were enriched for DNA, RNA and nucleic acid metabolic processes (Figure 4B). Proteins that were modified by both SUMO1 and SUMO2 were associated to various transcriptionrelated functions (Figure 4C). Ingenuity Pathway Analysis (IPA) confirmed the high-degree of involvement of both SUMO1 and SUMO2 in transcription and DNA/RNA processing (Figure 4D). IPA canonical pathway analysis revealed that proteins with SUMO1 sites were associated to cancer-related signaling pathways, while those with SUMO2 were linked to a variety of different mechanisms (Figure 4E). Comparison of all identified SUMOylated proteins in mpkCCD14 cells with a large-scale analysis of ubiquitylated proteins ${ }^{26}$ from mouse kidney, emphasized the importance of SUMOylation for transcription. Although the mpkCCD14 SUMO proteome (1124 SUMOylated proteins) is considerably smaller than the mouse kidney ubiquitin proteome (2725 ubiquitylated proteins), more transcription factors (TFs) are SUMOylated than ubiquitylated (Figure 4F). Approximately $15 \%$ of our mpkCCD14 SUMO proteome were TFs, whereas they only represent $1.2 \%$ of the mouse kidney ubiquitin proteome. Out of 165 identified SUMOylated TF genes, 70 of them were indicated by STRING analysis to interact with important water and electrolyte/solute transporters and channels (Table S4, Figure S2). These include Tsc22d3 (high 
confidence for regulating the epithelial sodium channel $\mathrm{ENaC}$ ), Gata3 and Nfat5 (high confidence for regulating the water channel AQP2), and Rxra, Ncor2 and Myc (high confidence for regulating the glucose transporter GLUT4).

Physiological regulation of SUMOylation in mpkCCD14 cells by aldosterone. Many of the actions of the mineralocorticoid aldosterone in the collecting duct are mediated through altered gene transcription via the mineralocorticoid receptor (MR). As SUMOylation in mpkCCD14 cells was in general associated with gene transcription, we hypothesized that protein SUMOylation in mpkCCD14 cells may change after aldosterone treatment. In line with this, treatment of mpkCCD-SUMO1 $1^{\text {T95K }}$ or mpkCCD-SUMO2 ${ }^{\text {T90K }}$ cells with aldosterone for $24 \mathrm{~h}$ resulted in redistribution of SUMO (Figure 5). SUMO1 labeling shifted from prominently around the cell nucleus into a more general distribution within the nucleus (Figure 5A and B). For SUMO2, aldosterone treatment resulted in a shift from a broad cytoplasmic labeling pattern to a more prominent perinuclear localization, with some nucleus labeling apparent (Figure 5C and D). It is unclear whether the immunolabeling represents a shift in localization of SUMOylated proteins after aldosterone, or increased SUMOylation within the specific compartments. To assess whether aldosterone altered SUMOylation levels of target proteins, the mpkCCD-SUMO1 ${ }^{\text {T95K }}$ or mpkCCD-SUMO2 ${ }^{\text {T90K }}$ cells were labelled for use in stable isotope in cell culture (SILAC) experiments, treated with aldosterone for $24 \mathrm{~h}$ and subject to LC-MS/MS. In general, the numbers of identified SUMOylated peptides after aldosterone treatment was very low (Table S5), especially between biological replicates. Whether this is due to limited effects of aldosterone to alter SUMOylation, or simply due to the technical limitations of the approach is unclear. In line with the former, only minor changes were observed in total SUMO1 or SUMO2 
levels following aldosterone treatment (relative to e.g. heat shock) when lysates from similar experiments were assessed by immunoblotting (Figure S3). Despite this, after rigorous data filtration, SUMOylation of the $\mathrm{H}^{+} /$oligopeptide co-transporter Pept2 (S/c15a2) by SUMO1 and SUMO2 was consistently increased 8-10 fold at K139, after aldosterone treatment (Table S5, Figure S4). Aldosterone did not alter S/c15a2 mRNA in wild-type mpkCCD14 cells (Figure 6A), suggesting that the effects are post-transcriptional. Furthermore, in a flag-tagged Pept2 transfected mpkCCD14 cell line (Pept2 antibodies unsuitable for western blots), Pept2 protein abundance was only increased approximately 2-fold by aldosterone (Figure 6B and C). Together, this data suggests that aldosterone increases SUMOylation levels of Pept2 directly.

\section{DISCUSSION}

Kidney epithelial cells have to rapidly adapt to an ever-changing external environment and respond to a variety of cellular stresses, including osmotic, genotoxic and oxidative stresses ${ }^{2}$. As SUMOylation is a mechanism that alters the functional properties of a variety of proteins, or alters protein expression, it allows a diverse response of cells to various forms of stress. It is therefore not surprising that SUMOylated proteins are involved in human renal diseases, including acute kidney injury ${ }^{18}$, renal cell carcinoma $^{19}$, and diabetic nephropathy ${ }^{20}$. However, understanding the role of protein SUMOylation in the kidney was hampered by a lack of information regarding the magnitude of SUMOylation targets in the kidney. Here we generated a SUMOylation landscape of renal cortical collecting duct (mpkCCD14) cells using proteomics as a first step to understanding the role of SUMOylation under physiological and pathophysiological conditions. 
The approach we used to generate a SUMOylation profile of mpkCCD14 cells involved expressing forms of SUMO in the cells that have minor modifications that allow differentiation of SUMO from other similar PTMs such as ubiquitylation and neddylation. Using this approach 1428/741 SUMO1 sites/proteins and 1957/971 SUMO2 sites/proteins were identified. Although these numbers are in line with the original study using this approach ${ }^{14}$ and could likely be increased with greater technical replicates and peptide fractionation ${ }^{17}$, they are much lower than numbers reported in newly developed approaches. For example, in 2014 Hendriks et al. reported $>4300 / 1600$ SUMO sites/proteins from Hela cells transfected with a highly mutated form of SUMO $2^{11}$. These numbers could be increased by technique optimization to $>40,000$ sites in transfected HeLa and U2OS (human bone osteosarcoma) cells ${ }^{13}$. Most recently over 14,000 endogenous SUMO2 sites were identified in human cells and mouse organs using a peptide level antibody enrichment method ${ }^{17}$. In the future, use of these newly developed techniques will be a powerful approach to study modulation of SUMOylated proteins in vivo in various disease models, including kidney disease.

Analysis of the SUMO proteome of mpkCCD-SUMO1 ${ }^{\text {T95K }}$ and mpkCCD-SUMO2 ${ }^{\text {T90K }}$ cells highlighted that there was a large overlap between SUMO1 and SUMO2 sites (40\%) and SUMOylated proteins (50\%), emphasizing substrate cross-reactivity between the isoforms. The greatest degree of overlap between the isoforms occurs on proteins involved in DNA/RNA transcription (and associated functions); whereas cell death pathways and DNA repair functions are extremely conserved for proteins modified by SUMO2. These results fit well with mouse models, where SUMO1 and SUMO3 deficient mice can survive due to compensation by SUMO2/3 or SUMO2 respectively 36,37 , whereas SUMO2 deficiency is lethal during embryogenesis $^{38}$. Further evidence of the importance of SUMOylation for modulating DNA/RNA transcription is supplied by the comparison with ubiquitylation, a modification known to play a 
role in gene control ${ }^{39,40}$, with substantially more SUMOylated than ubiquitylated TFs. Another observation from this study was that phosphorylation of SUMOylated proteins was enriched at positions -5 and +5 (relative to the targeted lysine), suggesting co-modification of the same protein and a potential importance of phosphorylation for SUMOylation to occur. A similar correlation was observed in mouse tissues ${ }^{13,17}$ and a phosphorylation-dependent SUMO modification (PDSM) motif that promotes SUMOylation has been proposed ${ }^{35}$. This motif occurs in several regulators of gene expression, including heat shock factors (HSFs) and TFs.

The mineralocorticoid aldosterone plays an essential role in the homeostatic control of plasma potassium and sodium levels and ultimately blood pressure ${ }^{41}$. Acting via the MR, aldosterone alters expression of various transporters or channels, including the epithelial sodium channel ( $\mathrm{ENaC}$ ) and the renal outer medullary potassium channel (ROMK), that mediate reabsorption of sodium and secretion of potassium in the kidney distal tubule. As SUMOylation appears to play a major role in modulating gene expression, it was logical to hypothesize that SUMOylation is linked to the actions of aldosterone. Indeed, both the mineralocorticoid receptor $(M R)^{42}$ and 11 Beta-hydroxysteroid dehydrogenase type $2^{43}$, the enzyme that limits access of glucocorticoids to the MR, are SUMOylated. Furthermore, aldosterone plays a major role in renal inflammatory responses in diabetic kidney disease and a role of SUMOylation has been proposed ${ }^{20,44}$. In line with an association between aldosterone and SUMOylation, in mpkCCD14 cells treated with aldosterone for $24 \mathrm{~h}$ SUMO labeling became more prominent in the perinuclear and nuclear regions, especially for SUMO2. However, attempts to quantify aldosterone induced changes in protein SUMOylation using SILAC and LC-MS/MS were less successful. There are several possible reasons for this. Firstly, the whole workflow for analysis of SUMOylation is technically challenging, which introduces small errors between replicates making statistical comparisons between groups difficult. Secondly, SUMOylated proteins 
themselves are so low in abundance that even minor differences between biological and technical replicates can introduce large differences in group comparisons. Thirdly, there are computational issues when using SILAC to quantify changes in SUMOylation as search engines do not allow dual mass adjustments of any single amino acid. Finally, contrary to our initial hypothesis it appears that in this cell model under the current conditions, aldosterone does not alter the SUMOylation landscape to the same degree as other cellular stresses such as heat shock. Despite these limitations in our SILAC study, SUMOylation of a specific $\mathrm{H}^{+}$/oligopeptide co-transporter, peptide transporter 2 (Pept2) $)^{45}$, was shown to be highly regulated by aldosterone. Although post-transcriptional effects of aldosterone to increase Pept2 protein were also observed ( 2 fold change), the magnitude of this change relative to the changes in SUMOylation (8-10 fold change) suggest that aldosterone modulates Pept2 SUMOylation directly. Further studies are required to assess whether the altered changes in protein abundance and SUMOylation levels are linked and the general role of SUMOylation for the function of this transporter.

In conclusion, this study generated a SUMOylation landscape of mpkCCD14 cells. A large number of the identified TFs that are SUMOylated are linked to the expression of proteins essential for water and electrolyte balance. This suggests that protein modification by SUMOylation is a mechanism within renal epithelial cells to modulate gene transcription under various physiological or pathophysiological conditions. However, extensive further studies are required to identify and characterize the physiological stimuli mediating these SUMOylation events.

\section{DISCLOSURE}

The authors declare no competing financial interest. 


\section{AUTHOR CONTRIBUTIONS}

R.A.F made the conception and initial design of the work. Q.W, T.S.A, L.C and R.A.F acquired data, analyzed and interpreted data. Q.W, T.S.A, and R.A.F drafted the manuscript. All authors critically revised manuscript for intellectual content, approved the final version of the manuscript and agree to be accountable for all aspects of the work.

\section{ACKNOWLEDGEMENTS}

Ronald T. Hay, Centre for Gene Regulation and Expression, University of Dundee is thanked for the pEFIRESpuro vectors containing N-terminal hexa-histidine tagged SUMO1 and SUMO2 ${ }^{14}$. We would like to acknowledge the technical assistance of Tina Drejer, Ahmed Abduljabar and Christian Westberg. Funding (to R.A.F) is provided by the Novo Nordisk Foundation (incl. Fabrikant Vilhelm Pedersen og Hustrus Mindelegat), the Lundbeck Foundation and the Danish Medical Research Council. R.A.F is further supported by the Leducq Foundation. Q.W. is supported by European Union Horizon 2020 Marie Skłodowska-Curie Individual Fellowship (Project No. 705682). 


\section{SUPPORTING INFORMATION}

The following supporting information is available free of charge at ACS website

http://pubs.acs.org

A complete and detailed description of the methods is enclosed in Supporting Information. Also included are:

Figure S1. Comparison of SUMOylation levels in the cell lines following various treatments that may enhance SUMOylation

Figure S2. STRING interaction scores

Figure S3. Immunoblotting for SUMO following $24 \mathrm{~h}$ and $48 \mathrm{~h}$ aldosterone $(1 \mu \mathrm{M})$ treatment

Figure S4. Example chromatogram of Pept2

Annex. Original immunoblots with areas utilized in figures highlighted

Table S1. Databases used for cross-reference

Table S2. Number of identified SUMO and total peptides/proteins

Table S3. mpkCCD14 cell SUMO proteome

Table S4. STRING interaction score between SUMOylated transcription factors and known renal transporters/channels

Table S5. SILAC quantification of SUMO sites/proteins between aldosterone-treated cells and vehicles 


\section{REFERENCES}

(1) Hay, R. T., SUMO: a history of modification. Mol. Cell. 2005, 18 (1), 1-12.

(2) Geiss-Friedlander, R.; Melchior, F., Concepts in sumoylation: a decade on. Nat. Rev. Mol. Cell. Biol. 2007, 8 (12), 947-956.

(3) Bohren, K. M.; Nadkarni, V.; Song, J. H.; Gabbay, K. H.; Owerbach, D., A M55V polymorphism in a novel SUMO gene (SUMO-4) differentially activates heat shock transcription factors and is associated with susceptibility to type I diabetes mellitus. J. Biol. Chem. 2004, 279 (26), 27233-27238.

(4) Liang, Y. C.; Lee, C. C.; Yao, Y. L.; Lai, C. C.; Schmitz, M. L.; Yang, W. M., SUMO5, a Novel Poly-SUMO Isoform, Regulates PML Nuclear Bodies. Sci. Rep. 2016, 6, 26509.

(5) Ma, X.; Yang, T.; Luo, Y.; Wu, L.; Jiang, Y.; Song, Z.; Pan, T.; Liu, B.; Liu, G.; Liu, J.; Yu, F.; He, Z.; Zhang, W.; Yang, J.; Liang, L.; Guan, Y.; Zhang, X.; Li, L.; Cai, W.; Tang, X.; Gao, S.; Deng, K.; Zhang, H., TRIM28 promotes HIV-1 latency by SUMOylating CDK9 and inhibiting P-TEFb. Elife. 2019, 8 .

(6) Flotho, A.; Melchior, F., Sumoylation: a regulatory protein modification in health and disease. Annu. Rev. Biochem. 2013, 82, 357-385.

(7) Matic, I.; Schimmel, J.; Hendriks, I. A.; van Santen, M. A.; van de Rijke, F.; van Dam, H.; Gnad, F.; Mann, M.; Vertegaal, A. C., Site-specific identification of SUMO-2 targets in cells reveals an inverted SUMOylation motif and a hydrophobic cluster SUMOylation motif. Mol. Cell. 2010, 39 (4), 641-652.

(8) Becker, J.; Barysch, S. V.; Karaca, S.; Dittner, C.; Hsiao, H. H.; Berriel Diaz, M.; Herzig, S.; Urlaub, H.; Melchior, F., Detecting endogenous SUMO targets in mammalian cells and tissues. Nat. Struct. Mol. Biol. 2013, 20 (4), 525-531.

(9) Hendriks, I. A.; Vertegaal, A. C., A comprehensive compilation of SUMO proteomics. Nat. Rev. Mol. Cell. Biol. 2016, 17 (9), 581-595.

(10) Witze, E. S.; Old, W. M.; Resing, K. A.; Ahn, N. G., Mapping protein post-translational modifications with mass spectrometry. Nat. Methods. 2007, 4 (10), 798-806.

(11) Hendriks, I. A.; D'Souza, R. C.; Yang, B.; Verlaan-de Vries, M.; Mann, M.; Vertegaal, A. C., Uncovering global SUMOylation signaling networks in a site-specific manner. Nat. Struct. Mol. Biol. 2014, 21 (10), 927-936.

(12) Tammsalu, T.; Matic, I.; Jaffray, E. G.; Ibrahim, A. F. M.; Tatham, M. H.; Hay, R. T., Proteome-wide identification of SUMO2 modification sites. Sci. Signal. 2014, 7 (323), rs2. 
(13) Hendriks, I. A.; Lyon, D.; Young, C.; Jensen, L. J.; Vertegaal, A. C.; Nielsen, M. L., Sitespecific mapping of the human SUMO proteome reveals co-modification with phosphorylation. Nat. Struct. Mol. Biol. 2017, 24 (3), 325-336.

(14) Tammsalu, T.; Matic, I.; Jaffray, E. G.; Ibrahim, A. F.; Tatham, M. H.; Hay, R. T., Proteomewide identification of SUMO modification sites by mass spectrometry. Nat. Protoc. 2015, 10 (9), 1374-1388.

(15) Lamoliatte, F.; McManus, F. P.; Maarifi, G.; Chelbi-Alix, M. K.; Thibault, P., Uncovering the SUMOylation and ubiquitylation crosstalk in human cells using sequential peptide immunopurification. Nat. Commun. 2017, 8, 14109.

(16) Lumpkin, R. J.; Gu, H.; Zhu, Y.; Leonard, M.; Ahmad, A. S.; Clauser, K. R.; Meyer, J. G.; Bennett, E. J.; Komives, E. A., Site-specific identification and quantitation of endogenous SUMO modifications under native conditions. Nat. Commun. 2017, 8 (1), 1171.

(17) Hendriks, I. A.; Lyon, D.; Su, D.; Skotte, N. H.; Daniel, J. A.; Jensen, L. J.; Nielsen, M. L., Sitespecific characterization of endogenous SUMOylation across species and organs. Nat. Commun. 2018, 9 (1), 2456.

(18) Guo, C.; Wei, Q.; Su, Y.; Dong, Z., SUMOylation occurs in acute kidney injury and plays a cytoprotective role. Biochim. Biophys. Acta. 2015, 1852 (3), 482-489.

(19) Dong, B.; Gao, Y.; Kang, X.; Gao, H.; Zhang, J.; Guo, H.; You, M. J.; Xue, W.; Cheng, J.; Huang, Y., SENP1 promotes proliferation of clear cell renal cell carcinoma through activation of glycolysis. Oncotarget. 2016, 7 (49), 80435-80449.

(20) Li, O.; Ma, Q.; Li, F.; Cai, G. Y.; Chen, X. M.; Hong, Q., Progress of small ubiquitin-related modifiers in kidney diseases. Chin. Med. J (Engl). 2019, 132 (4), 466-473.

(21) Yu, M. J.; Miller, R. L.; Uawithya, P.; Rinschen, M. M.; Khositseth, S.; Braucht, D. W.; Chou, C. L.; Pisitkun, T.; Nelson, R. D.; Knepper, M. A., Systems-level analysis of cell-specific AQP2 gene expression in renal collecting duct. Proc. Natl. Acad. Sci. U. S. A. 2009, 106 (7), 2441-2446.

(22) Wu, Q.; Moeller, H. B.; Stevens, D. A.; Sanchez-Hodge, R.; Childers, G.; Kortenoeven, M. L. A.; Cheng, L.; Rosenbaek, L. L.; Rubel, C.; Patterson, C.; Pisitkun, T.; Schisler, J. C.; Fenton, R. A., CHIP Regulates Aquaporin-2 Quality Control and Body Water Homeostasis. J. Am. Soc. Nephrol. 2018, 29 (3), 936-948.

(23) Yde, J.; Keely, S.; Wu, Q.; Borg, J. F.; Lajczak, N.; O'Dwyer, A.; Dalsgaard, P.; Fenton, R. A.; Moeller, H. B., Characterization of AQPs in Mouse, Rat, and Human Colon and Their Selective Regulation by Bile Acids. Front. Nutr. 2016, 3, 46. 
(24) Moeller, H. B.; Praetorius, J.; Rutzler, M. R.; Fenton, R. A., Phosphorylation of aquaporin-2 regulates its endocytosis and protein-protein interactions. Proc. Natl. Acad. Sci. U. S. A. 2010, $107(1), 424-429$.

(25) Perez-Riverol, Y.; Csordas, A.; Bai, J.; Bernal-Llinares, M.; Hewapathirana, S.; Kundu, D. J.; Inuganti, A.; Griss, J.; Mayer, G.; Eisenacher, M.; Perez, E.; Uszkoreit, J.; Pfeuffer, J.; Sachsenberg, T.; Yilmaz, S.; Tiwary, S.; Cox, J.; Audain, E.; Walzer, M.; Jarnuczak, A. F.; Ternent, T.; Brazma, A.; Vizcaino, J. A., The PRIDE database and related tools and resources in 2019: improving support for quantification data. Nucleic Acids Res. 2019, 47 (D1), D442-D450.

(26) Wagner, S. A.; Beli, P.; Weinert, B. T.; Scholz, C.; Kelstrup, C. D.; Young, C.; Nielsen, M. L.; Olsen, J. V.; Brakebusch, C.; Choudhary, C., Proteomic analyses reveal divergent ubiquitylation site patterns in murine tissues. Mol. Cell. Proteomics. 2012, 11 (12), 1578-1585.

(27) Chan, W. K.; Zhang, H.; Yang, J.; Brender, J. R.; Hur, J.; Ozgur, A.; Zhang, Y., GLASS: a comprehensive database for experimentally validated GPCR-ligand associations. Bioinformatics. 2015, 31 (18), 3035-3042.

(28) Zhang, H. M.; Liu, T.; Liu, C. J.; Song, S.; Zhang, X.; Liu, W.; Jia, H.; Xue, Y.; Guo, A. Y., AnimalTFDB 2.0: a resource for expression, prediction and functional study of animal transcription factors. Nucleic Acids Res. 2015, 43 (Database issue), D76-81.

(29) Gao, T.; Liu, Z.; Wang, Y.; Cheng, H.; Yang, Q.; Guo, A.; Ren, J.; Xue, Y., UUCD: a familybased database of ubiquitin and ubiquitin-like conjugation. Nucleic Acids Res. 2013, 41 (Database issue), D445-451.

(30) Lee, J. W.; Chou, C. L.; Knepper, M. A., Deep Sequencing in Microdissected Renal Tubules Identifies Nephron Segment-Specific Transcriptomes. J. Am. Soc. Nephrol. 2015, 26 (11), 26692677.

(31) Szklarczyk, D.; Franceschini, A.; Wyder, S.; Forslund, K.; Heller, D.; Huerta-Cepas, J.; Simonovic, M.; Roth, A.; Santos, A.; Tsafou, K. P.; Kuhn, M.; Bork, P.; Jensen, L. J.; von Mering, C., STRING v10: protein-protein interaction networks, integrated over the tree of life. Nucleic Acids Res. 2015, 43 (Database issue), D447-452.

(32) Manza, L. L.; Codreanu, S. G.; Stamer, S. L.; Smith, D. L.; Wells, K. S.; Roberts, R. L.; Liebler, D. C., Global shifts in protein sumoylation in response to electrophile and oxidative stress. Chem. Res. Toxicol. 2004, 17 (12), 1706-1715.

(33) Ayaydin, F.; Dasso, M., Distinct in vivo dynamics of vertebrate SUMO paralogues. Mol. Biol. Cell. 2004, 15 (12), 5208-5218. 
(34) Wisniewski, J. R., Filter-Aided Sample Preparation for Proteome Analysis. Methods Mol. Biol. 2018, 1841, 3-10.

(35) Hietakangas, V.; Anckar, J.; Blomster, H. A.; Fujimoto, M.; Palvimo, J. J.; Nakai, A.; Sistonen, L., PDSM, a motif for phosphorylation-dependent SUMO modification. Proc. Natl. Acad. Sci. U. S. A. 2006, 103 (1), 45-50.

(36) Zhang, F. P.; Mikkonen, L.; Toppari, J.; Palvimo, J. J.; Thesleff, I.; Janne, O. A., Sumo-1 function is dispensable in normal mouse development. Mol. Cell. Biol. 2008, 28 (17), 5381-5390.

(37) Evdokimov, E.; Sharma, P.; Lockett, S. J.; Lualdi, M.; Kuehn, M. R., Loss of SUMO1 in mice affects RanGAP1 localization and formation of PML nuclear bodies, but is not lethal as it can be compensated by SUMO2 or SUMO3. J. Cell. Sci. 2008, 121 (Pt 24), 4106-4113.

(38) Wang, L.; Wansleeben, C.; Zhao, S.; Miao, P.; Paschen, W.; Yang, W., SUMO2 is essential while SUMO3 is dispensable for mouse embryonic development. EMBO Rep. 2014, 15 (8), 878885.

(39) Salghetti, S. E.; Caudy, A. A.; Chenoweth, J. G.; Tansey, W. P., Regulation of transcriptional activation domain function by ubiquitin. Science. 2001, 293 (5535), 1651-1653.

(40) Muratani, M.; Tansey, W. P., How the ubiquitin-proteasome system controls transcription. Nat. Rev. Mol. Cell. Biol. 2003, 4 (3), 192-201.

(41) Thomas, W.; Harvey, B. J., Mechanisms underlying rapid aldosterone effects in the kidney. Annu. Rev. Physiol. 2011, 73, 335-357.

(42) Yokota, K.; Shibata, H.; Kurihara, I.; Kobayashi, S.; Suda, N.; Murai-Takeda, A.; Saito, I.; Kitagawa, H.; Kato, S.; Saruta, T.; Itoh, H., Coactivation of the N-terminal transactivation of mineralocorticoid receptor by Ubc9. J. Biol. Chem. 2007, 282 (3), 1998-2010.

(43) Jimenez-Canino, R.; Lorenzo-Diaz, F.; Odermatt, A.; Bailey, M. A.; Livingstone, D. E. W.; Jaisser, F.; Farman, N.; Alvarez de la Rosa, D., 11beta-HSD2 SUMOylation Modulates CortisolInduced Mineralocorticoid Receptor Nuclear Translocation Independently of Effects on Transactivation. Endocrinology. 2017, 158 (11), 4047-4063.

(44) Briet, M.; Schiffrin, E. L., Aldosterone: effects on the kidney and cardiovascular system. Nat. Rev. Nephrol. 2010, 6 (5), 261-273.

(45) Shen, H.; Smith, D. E.; Yang, T.; Huang, Y. G.; Schnermann, J. B.; Brosius, F. C., 3rd, Localization of PEPT1 and PEPT2 proton-coupled oligopeptide transporter mRNA and protein in rat kidney. Am. J. Physiol. 1999, 276 (5), F658-665. 


\section{Figure legends}

Figure 1. Characterization of SUMOylation in mpkCCD14 cells. A-C) Wildtype or SUMO overexpressing mpkCCD14 cells in control conditions $\left(37^{\circ} \mathrm{C} /\right.$ vehicle (Veh)) or with heat shock $\left(43^{\circ} \mathrm{C}\right) / \mathrm{MG} 132+\mathrm{PR} 619$ (M+P). Immunoblots were probed with SUMO1, SUMO2, 6xHis, $\alpha$-Actin (Act) or Proteasome 20s (P20s) antibodies. D-F) Confocal laser scanning microscopy images of SUMO immunolabelling with SUMO1, SUMO2, or 6xHis antibodies in mpkCDD14-WT, mpkCCD-SUMO1 ${ }^{\text {T95K }}$ or mpkCCD-SUMO2 ${ }^{\text {T90K }}$ cells under control conditions or after heat shock. For image acquisition the microscope gain levels were set higher for the mpkCDD14-WT cells.

Figure 2. Workflow of SUMOylation analysis. A) Example of purification of histidine tagged proteins with nickel-nitrilotriacetic acid (Ni-NTA) agarose beads in mpkCDD14-WT and mpkCCD-SUMO1 ${ }^{\text {T95K }}$ cells. Loading order: Lysate input (Input), flow-through (FT), First-Fifth washes (W1-W5) and the purified protein elution (Elution). The Coomassie blot shows equal amount of input proteins in mpkCDD14-WT and mpkCCD-SUMO1 ${ }^{\text {T95K }}$ cells, whereas SUMO1 is enriched in the elution from transfected cells. B) Schematic overview of the experimental workflow (created with BioRender.com).

Figure 3. The SUMO landscape of mpkCCD14 cells. A) SUMO1 and SUMO2 site counts and B) protein counts from mpkCCD-SUMO1T95K or mpkCCD-SUMO2T90Kcells. C) Site overlap between SUMO1 and SUMO2 D) Overlap between proteins modified with SUMO1 and SUMO2. E) Distribution of singleSUMOylated and multi-SUMOylated proteins of SUMO1 and F) SUMO2. G) Complementary nature of different methods to assess SUMO2 sites. Sites in this study are compared to the mouse kidney endogenous SUMO2 sites17. H) Similar comparison for SUMO2 proteins. I) For the SUMOylated site a KxE consensus motif was observed for the acceptor lysine site and a reverse KxD/E motif. J) Schematic overview of SUMOylation and phosphorylation distribution. SUMOylated sites were from this study, while all known phosphorylation sites from PhosphoSitePlus were taken into account. SUMOylated lysines are at position 0 , with spacing indicating the relative position of phosphorylation. K) Similar as (J), but with both SUMOylation and phosphorylation sites only from this study. 
Figure 4. Assessment of the mpkCCD14 SUMO proteome. Gene ontology (GO) analysis of A) SUMO1 and B) SUMO2 exclusive proteins suggest that SUMO1 and SUMO2 proteins have different cellular localizations and functions. C) SUMO1 and SUMO2 proteins are associated with transcription-related functions. D) IPA "diseases and biofunctions" analysis confirmed the high-degree involvement of SUMO1 and SUMO2 in transcription. E) IPA "canonical pathways" analysis indicates that SUMO1 proteins have more distinct functions, whereas SUMO2 is implicated in a larger number of cellular processes. F) Comparison of SUMOylated or ubiquitylated proteins of various classes. Transcription factors were overrepresented in the transcription factor class, whereas transporters and channels were much more likely to be ubiquitylated.

Figure 5. Redistribution of SUMO following aldosterone. Confocal laser scanning microscopy images of SUMO immunolabelling in mpkCCD-SUMO1T95K or mpkCCD-SUMO2T9OKcells treated with vehicle (DMSO) or aldosterone (aldo) for $24 \mathrm{~h}$. In mpkCCD-SUMO1T95K was predominantly perinuclear in control conditions (A), but appeared to be more within the nucleus after aldosterone (B). In mpkCCDSUMO2T90K cells SUMO was distributed in the cytoplasm in control conditions (C), but was distinctly perinuclear following aldosterone (D).

Figure 6. Aldosterone increases Pept2 levels post-transcriptionally. A) RT-qPCR showed no significant changes in S/c15a2 mRNA (Pept2) in mpkCCD14-WT cells after aldosterone treatment. B) Representative western blot of Flag-tagged Pept2 transfected mpkCCD14 cells under control conditions or after treatment with aldosterone for $24 \mathrm{~h}$. C) Western blot quantification data from three experiments. $* * * *$ $\mathrm{p}<0.0001$. Veh $=$ vehicle, Aldo $=$ aldosterone $(1 \mu \mathrm{M})$. 
A)

mpkCCD14-WT

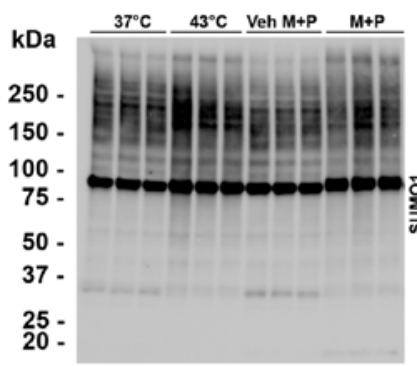

$50 \cdot------\square-\square$

B) mpkCCD-sumo1 ${ }^{\text {T9sk }}$

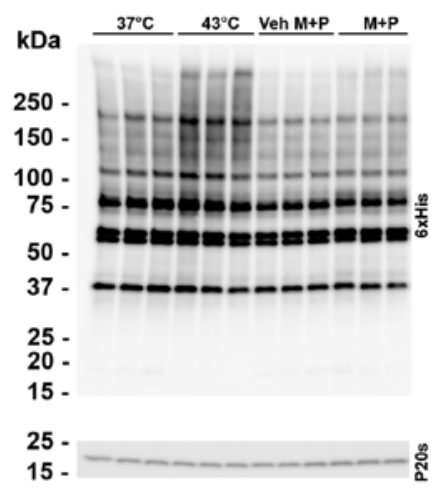

C) $\quad$ mpkCCD-sUMO2 $2^{\text {T9ok }}$

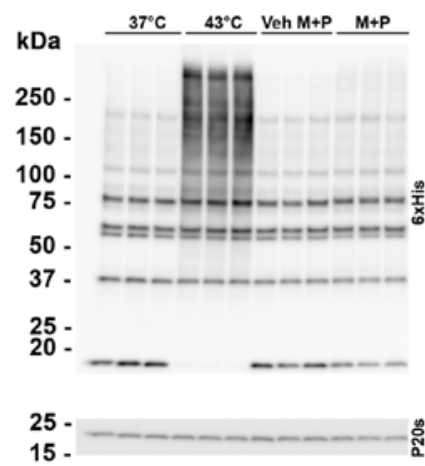

D)

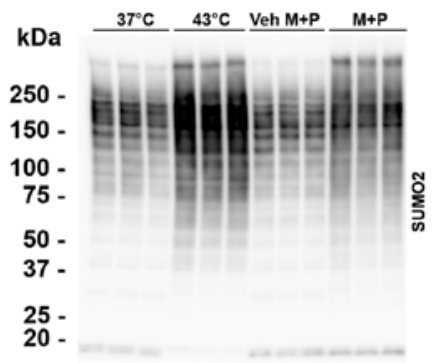

E)
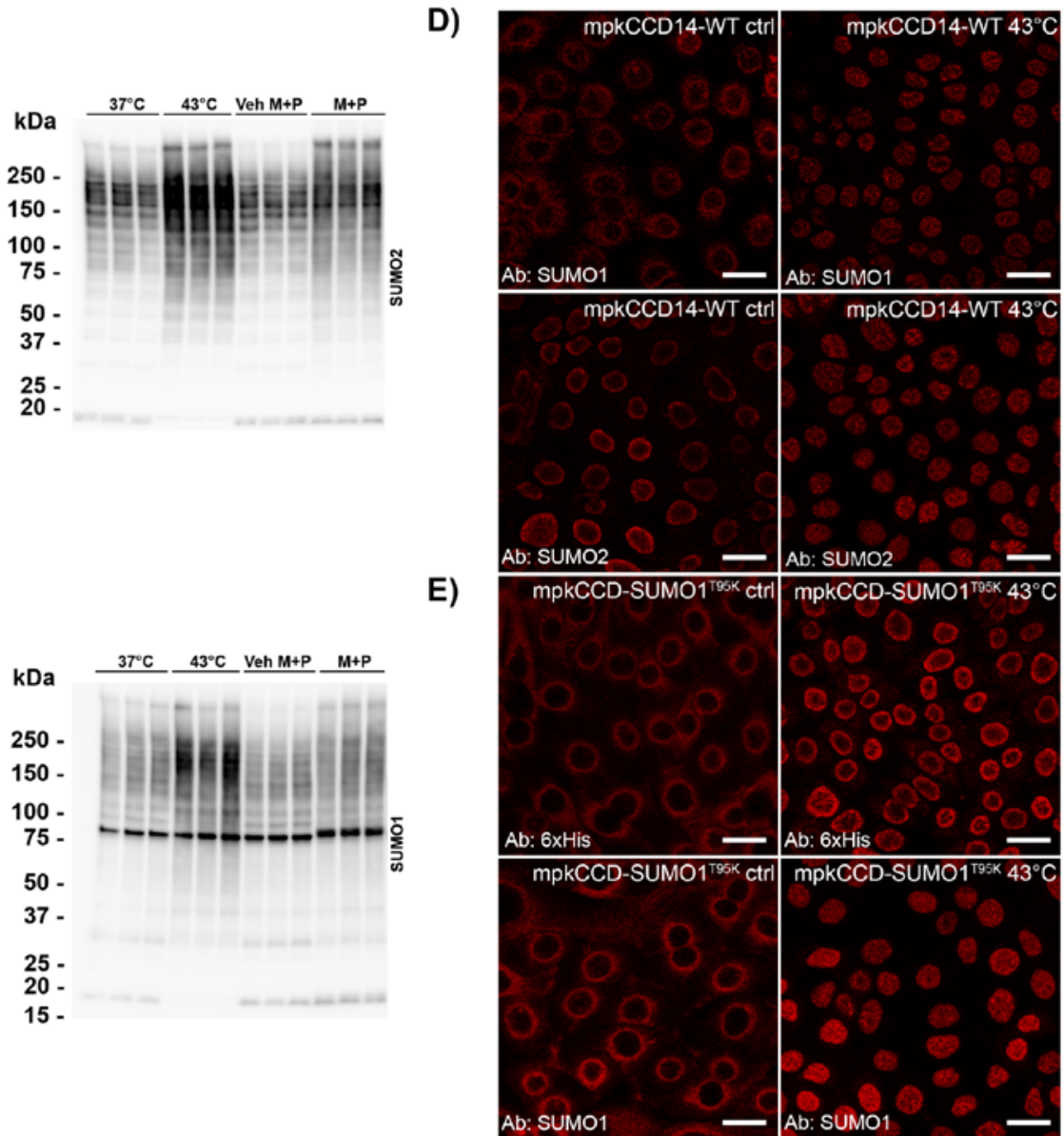

F)
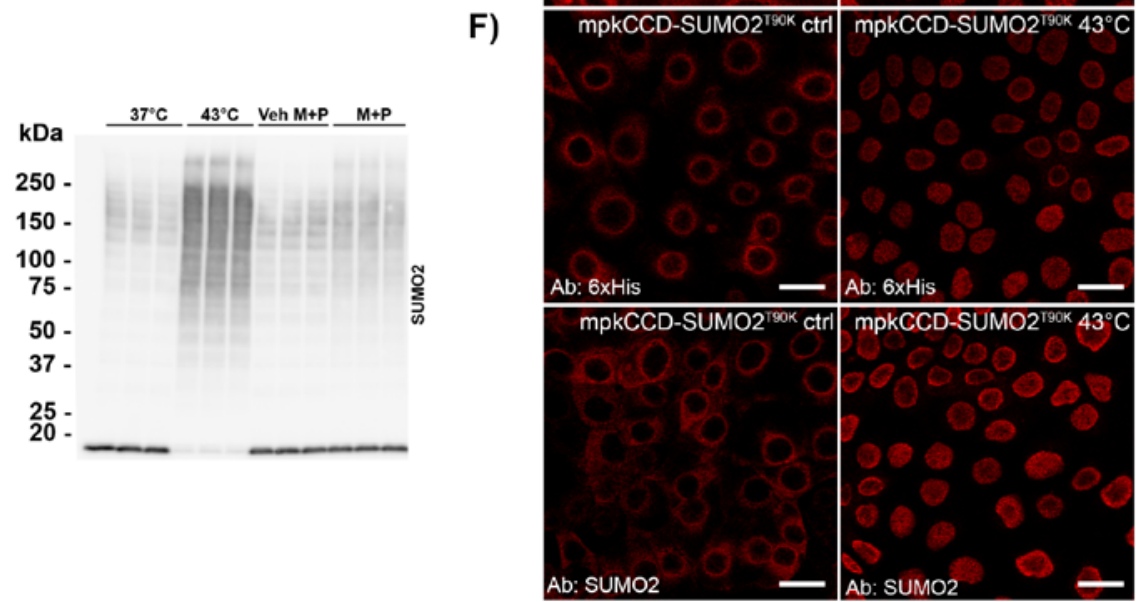

Figure 1 

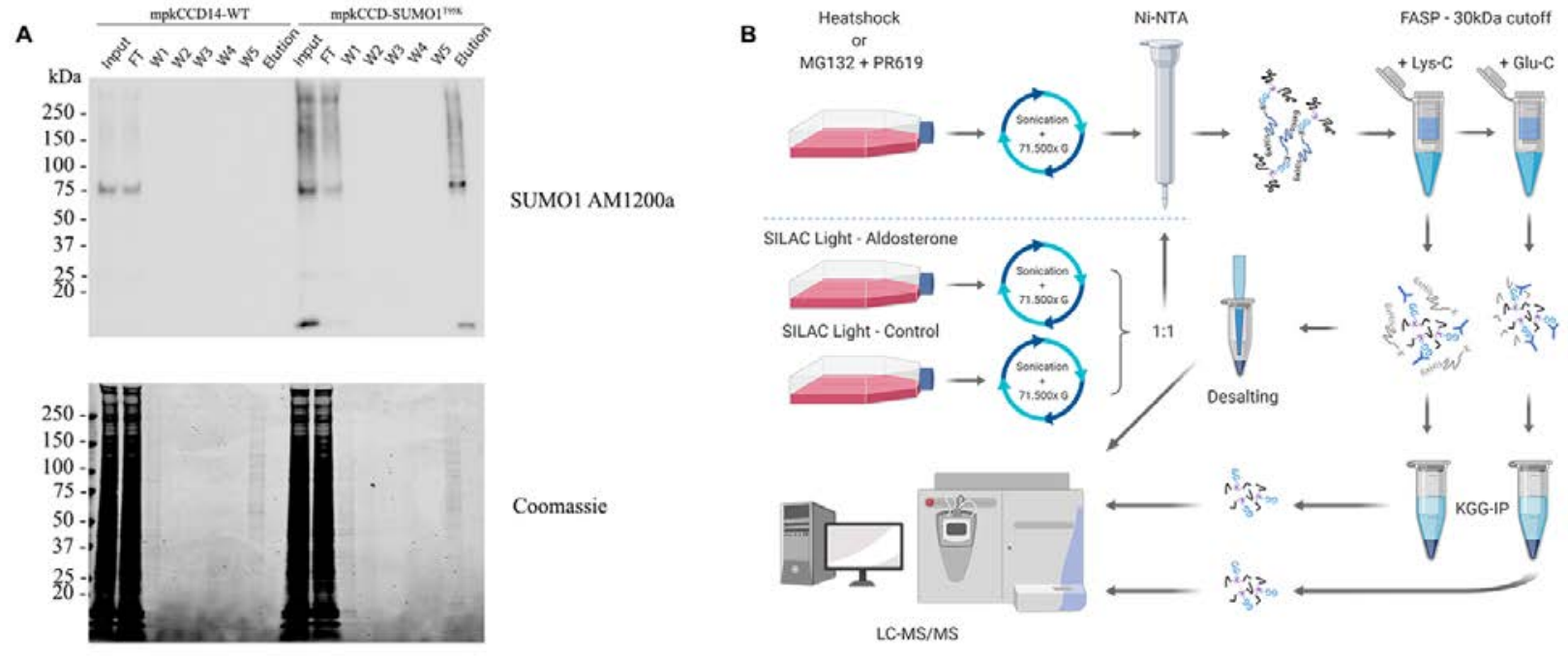

Figure 2 
A

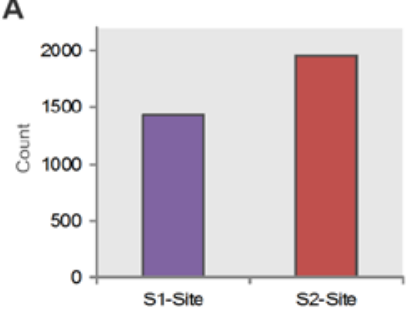

E

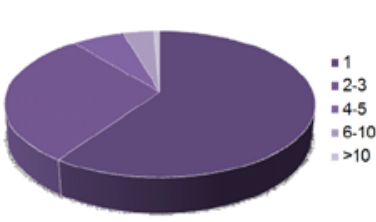

B

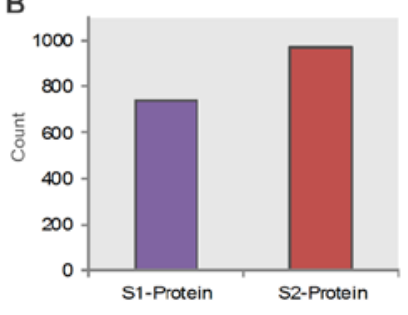

C
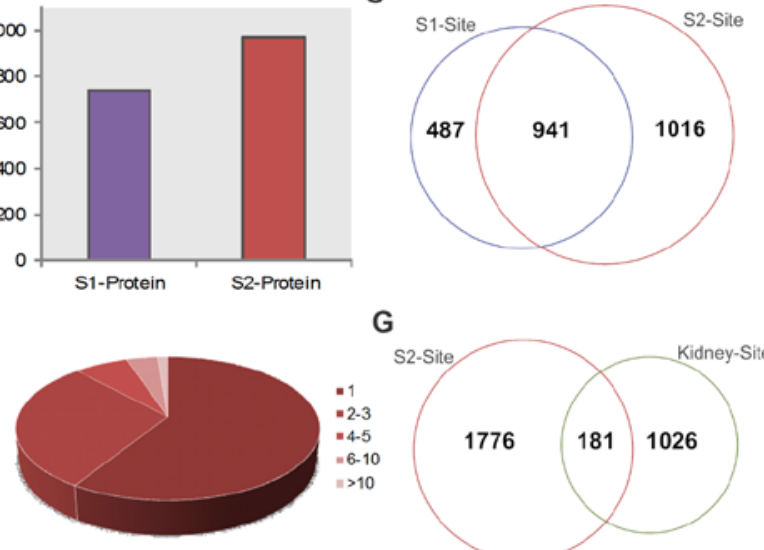

G

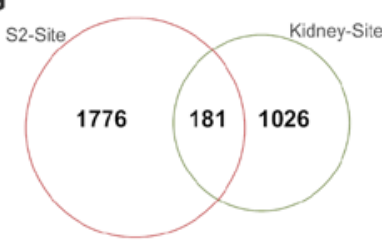

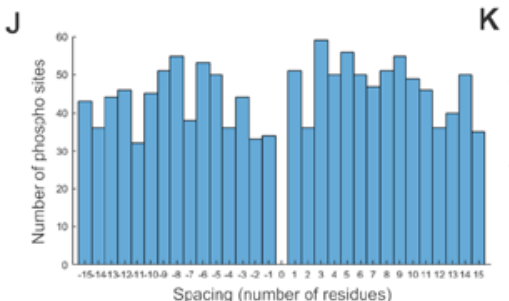

K

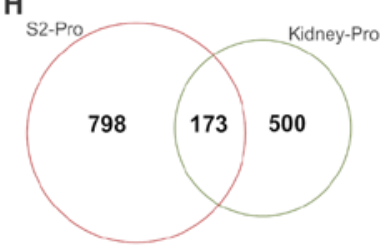

D
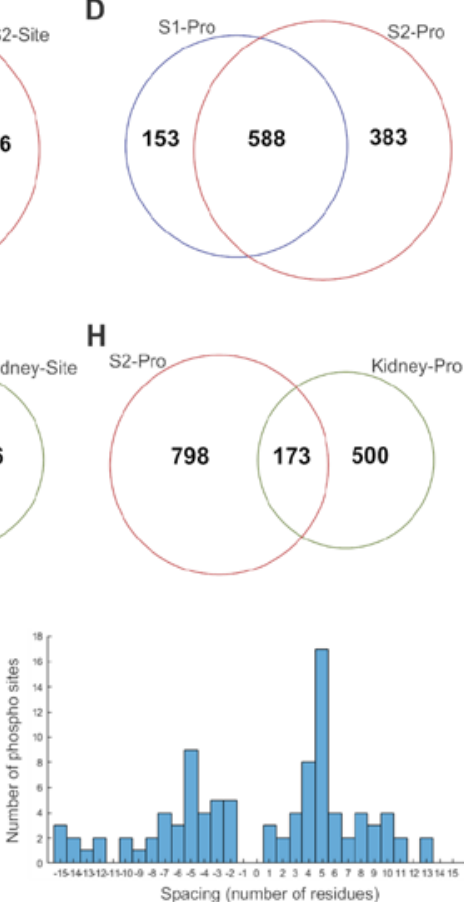

Figure 3 
A

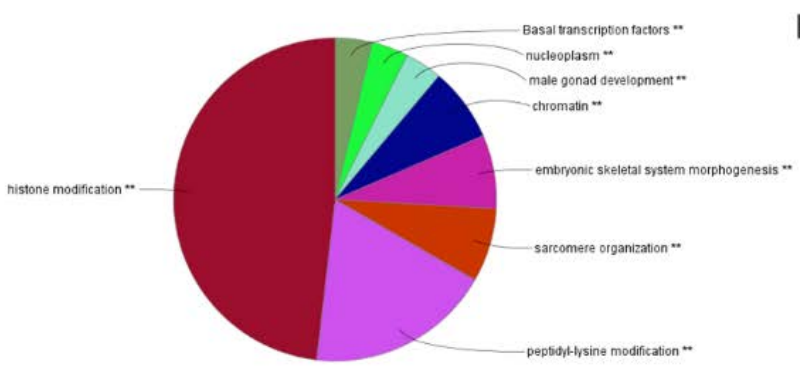

C

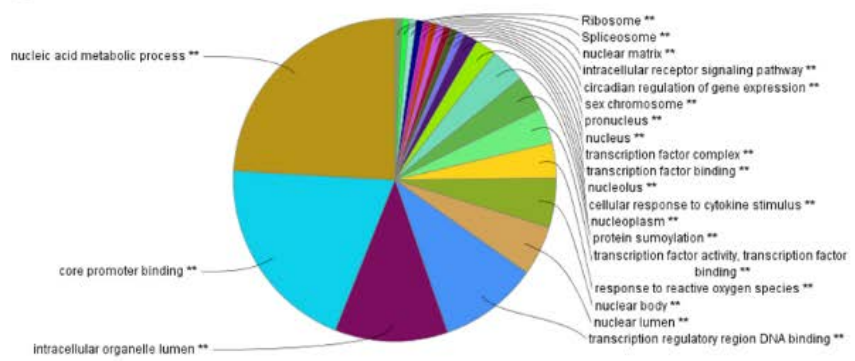

B

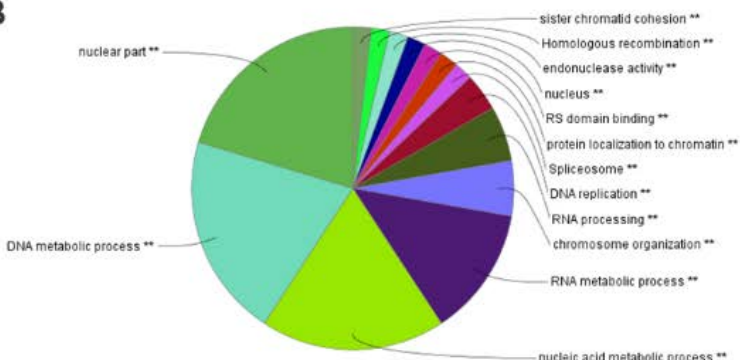

D

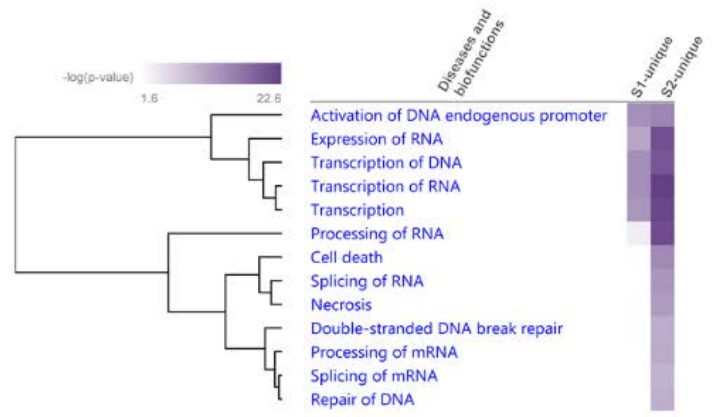

$F$

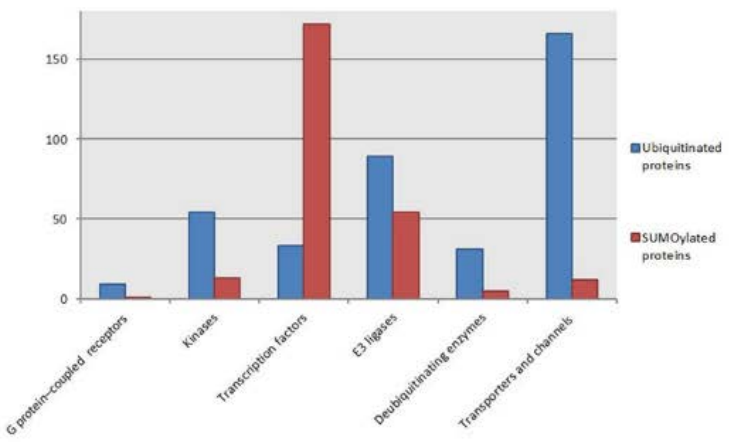

Figure 4 


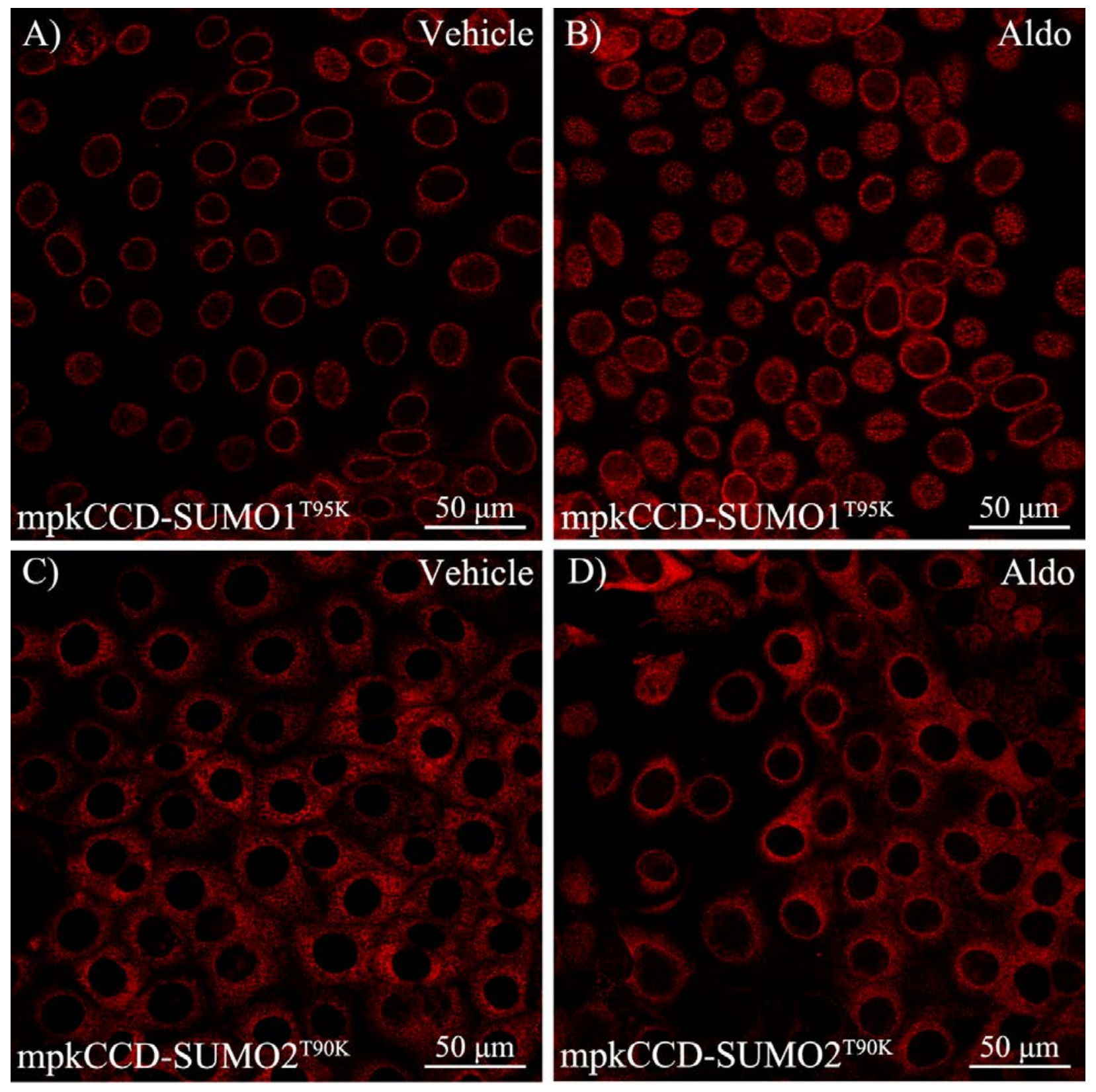

Figure 5 
A)

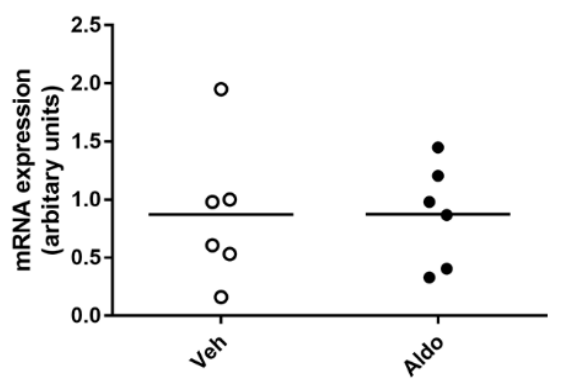

B)

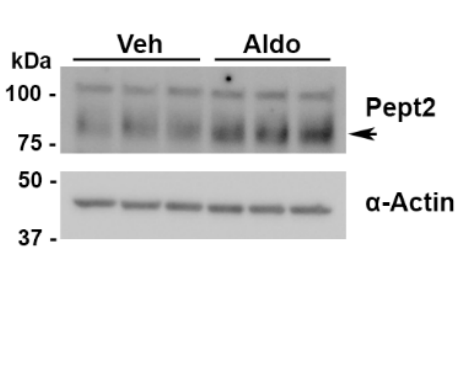

C)
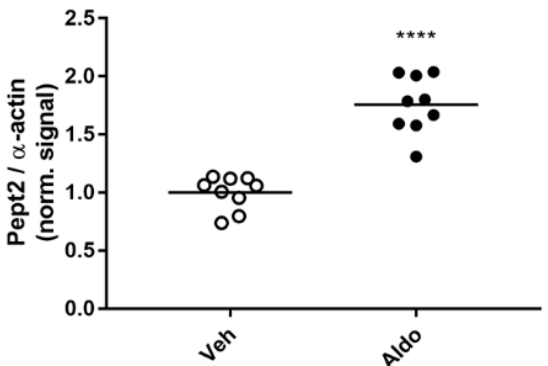

Figure 6 